\title{
Recent lessons learnt from the outbreak of brucellosis in Dekemhare, Anseba, Eritrea .
}

Abraham Kebedom1, Tadesse Teclebirhan1, Tsegai Tekest2, Berhane Zekarias3, Jacob Mufunda1, Andemariam Gebremichael1. Institutional Affiliation

1 Orotta School of Medicine, Asmara, Eritrea

2 Ministry of Health, Eritrea, Surveillance Office, Anseba Zone

3 Orotta National Referral Medical and Surgical Hospital, Asmara, Eritrea

Correspondence to be sent to: Tadesse Teclebirhan Orotta School of Medicine P.O. Box $10549 \quad$ Email:Tadesse09@gmail.com

Abstract

Brucellosis is an acute to chronic zoonotic disease which is endemic in some tropical developing countries with profound impact on infected animals and people. The study examined some of the risk factors for brucellosis following outbreak of the disease in Anseba, a region in Eritrea. Twenty three patients from Dekemhare, 2 of whom were admitted with confirmed diagnosis of brucellosis in Orotta National Referral Medical and Surgical Hospital in Asmara while the other 21, two thirds of whom confirmed positive for brucellosis according to the Rose Bengal slide agglutination test, sought services in local health facilities in Dekemhare following an outbreak of the disease in May 2008. More than $80 \%$ of the patients mostly students, consumed un-boiled milk. Two thirds of the animals in the area were sick but less than $20 \%$ received veterinary consultations with half of them testing positive for brucellosis. This report links contraction of brucellosis to human through contact with infected animals and drinking of un-boiled milk. Appropriate health promotion focusing on young people and targeting preventive measures such as regular use of veterinary services and consumption of boiled milk complemented by monitoring of brucellosis through integrated disease surveillance and response in Eritrea may cost effectively prevent brucellosis.Key words: PMTCT, LQAS

Keywords: Zoonosis, brucellosis, Eritrea, veterinary services

\section{Introduction}

Brucellosis ranges from an acute infectious disease to a chronic debilitating zoonotic infection that is transmitted by animals to humans and not from human to human. Direct contact with infected animals or consumption of un-boiled milk and soft dairy products are the primary routes of transmission of brucellosis. The disease is prevalent in the tropical developing countries where it was shown to coexist with malaria, with workers in the veterinary service workforce, dairy farm workers, and nomadic pastoralists being population groups at highest risk 1.

Brucellosis is one of the most prevalent and yet most under-diagnosed zoonotic diseases in equal measure, with profound human and animal economic adversity2. The pathogenic types of this intracellular organism are brucella melitensis, brucella suis, and brucella arbotus presenting major diagnostic challenges because of genomic variances.

The acute clinical presentation of the disease continues to pose a clinical diagnosis conundrum. Acute forms of Brucellosis manifest with a myriad spectrum of clinical presentations in some cases characterized by fever, chills, sweating, malaise, weakness and various types of pains occur in man making the disease qualify to membership of a clique of diseases of "champions of mimicry"; tuberculosis, malaria, syphilis and HIV/AIDS 3.

Rose Bengal slide and laboratory confirmatory tube agglutination tests are still the main but inconclusive diagnostic modalities. Blood cultures are considered to be the golden standard although they carry a low bacterial yield giving rise to false negatives/delay of diagnosis and development of the chronic stage of the disease 4,5. Brucellosis, like tuberculosis, is a chronic granulomatous infection caused by intracellular bacteria that requires combined, protracted broad spectrum antibiotic therapy. The disease causes formidable morbidity as well as a considerable loss of productivity in animal husbandry in the developing world 2.

Elusive accurate clinical diagnosis coupled with inconclusive laboratory confirmation and high treatment failure with high relapse rates guarantee disease chronicity and consequent high morbidity and related mortality. A recent outbreak of brucellosis in one region of Eritrea prompted the conduct of this study to determine the extent of the disease and risk factors for contracting the disease and identify the vulnerable groups in order to institute control measures.

\section{Methods}

A cross-sectional study was conducted to identify risk factors and identify vulnerable groups for contracting brucellosis infection in Dekemhare in Anseba, Eritrea. Data were collected from 23 patients 17 of whom were Rose Bengal slide test positive for the disease while the other 6 patients with symptoms suggestive of the disease were negative. A questionnaire was administered to all the 23 patients to gather data on socio-demographic, life style, factors associated with the disease and the management of the disease. Blood samples from the 23 patients were tested for brucellosis using the Rose Bengal tests.

\section{Results}

The mean age was 20.8 years with $74 \%$ of patients 
were students (Table 1).

Table 1: Brucellosis human cases distribution by age

\begin{tabular}{c|c|c} 
No. & Age in Years & Frequency \\
1. & $5-10$ & 1 \\
2 & $11-20$ & 13 \\
3 & $21-30$ & 7 \\
4 & $>30$ & 2
\end{tabular}

All of patients raised domestic animals like goats and cattle and lived with them in their homes. Two thirds of the animals faced abnormal health conditions for example some of them had history of abortions, but only $17 \%$ received veterinary consultations and were tested for brucellosis with $50 \%$ turning out to be positive in Dekemhare where 14 of the human cases of brucellosis came from (Figure 1).

\section{Figure 1}

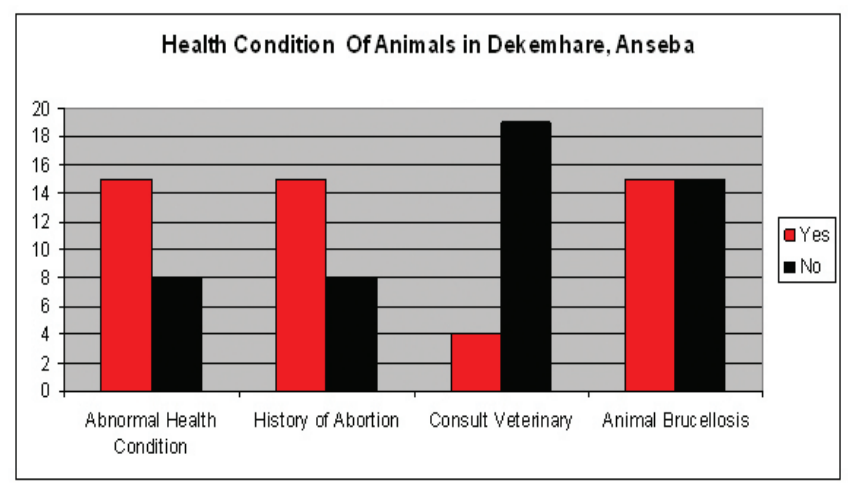

Out of the 23 patients 16 were positive for brucellosis and 7 were negative (Table 2). In Dekizeru 2 cases of animal brucellosis and 2 cases of human brucellosis were found. $57 \%$ of the cases had contact with animal fluids and carcasses (Table 2).

\begin{tabular}{l|l|l|}
$\begin{array}{l}\text { Table2: Human Brucellosis case and contact with } \\
\text { animals }\end{array}$ & Yes & No \\
History of contact with animal fluids & 13 & 10 \\
Human Brucellosis & 16 & 7
\end{tabular} \mid

All the patients used un-boiled milk for yogurt but cooked their meat properly before consumption.

All the patients were treated for brucellosis using a combination of antibiotics typically doxycycline $200 \mathrm{mg} /$ day orally for 45 days and rifampicin 600-900 $\mathrm{mg} /$ day for 2 weeks. 19 patients (i.e.83\%) of them fully recovered on this treatment regimen. Out of the 16 brucellosis positive cases 15 were improved (i.e. 94\%).

\section{Discussion}

A follow up study to the outbreak of 2 confirmed cases of brucellosis who surfaced at the national referral hospital identified risk factors for the two cases and exposed 21 other cases presenting with similar problems. No blood cultures were done but based on
Rose Bengal slide agglutination test, the sero-positivity of patients was more than $60 \%$. The accuracy of the clinical level of diagnosis was high because most of the diagnosed recovered on treatment suggesting they were either diagnosed early with recovery of more than $80 \%$ of all cases and more than $90 \%$ for positive cases or that the case management was optimal, whichever applied this was strength to improve on.

Previous studies have identified population subgroups at risk as being workers in veterinary services, or pastoralist, however in this study the majority was students which potentially makes it easier to prevent through targeted health promotion focusing on schools 1 .

In this study the majority of patients were students which are similar to findings from other studies 6 but different from others where most of the cases were among abattoir works especially those who slit the throats of animals or cleaned meat of slaughtered animals7. The use of unbolied milk was the major risk factor consistent with findings from other studies which incriminated the habit of drinking fresh goat milk from original source 6 .

Concurrent infection of people residing in similar environmental settings and sharing food patterns poses a major policy management challenge. This compounded by the wide range of symptoms which index cases may present with. Screening the cases and contacts with Rose Bengal slide Test and confirmatory laboratory tube tests may yield positive cases which may be asymptomatic and technically requiring follow up8. In our case contact was done a process which could have led to underreporting. Other factors linked to underreporting are under-diagnosing, poor disease surveillance, and weak policies and strategies9. The differentiation of passive from active brucellosis is difficult using the standard Rose Bengal test especially in endemic regions that require the use of PCR, a technique not always considered cost effective in resource poor countries where there are many other competing priorities 10 .

In our setting, once the brucellosis was identified an inter-ministerial team from Health and Agriculture headed by the IDSR of the $\mathrm{MOH}$ of Anseba region acted fast and made the standard way to treat the cases and a mass sensitization about brucellosis were done in all sub zones. At the same time the MOA was informed and the animals of the village were tested for brucellosis and $50 \%$ of them were positive. From the study it was found that this disease is mainly from the infected animals and the people's level of awareness towards brucellosis was poor. But once they were sensitized they are seeking the medical help.

The medical treatment according to the WHO guidelines was very efficacious with more than $80 \%$ of all cases and $90 \%$ of the positive cured within the recommended treatment duration. This positive outcome could be attributed to early diagnosis or aggressive case management or both. According to the $\mathrm{MOH}$ (IDSR unit) brucellosis is not in the list of disease of public health importance. It is not an emergency to treat it but its effect on the long run 
is more than an emergency. It could be explained by simple reduction in dairy product of country leading to poor socioeconomic level, especially to the farmers and the malnutrition of children is an alarm more than an emergency. It has to be underlined that the main stay of veterinary prevention is a national commitment to testing and taking the appropriate measures, otherwise only treating the humans is a temporary action. It is also concluded that the close contact with the infected animals and the use of unboiled milk for yogurt were found to be the main risks to acquire the zoonotic disease brucellosis. Lack of implementation of the recommendations from previous studies that highlighted need for inclusion of the disease in IDSR is another area worthy of strengthening.

\section{Acknowledgment}

We are grateful to Orotta School of Medicine, Research and Human Resource Development of Ministry of Health. Thanks to $\mathrm{MOH}$ (IDSR unit) Dr. Fikremariam Ghillamichael and Tesfalem Medhanie. Last but not least thanks to all the villagers involved in this study and Dekemhare village administrator.

\section{References:}

1. Omer MK, Aseffaw T, Skjerve E, Tekleghiorgis T, Woldehaimant Z. Prevalence of antibodies to Brucella spp. and risk factors related to high-risk occupational groups in Eritrea. Epidemiol Infect. 2002; 129: 85-91.
2. Young EJ. An overview of human brucellosis. Clin Infect Dis 1995; 21:283-9.

3. Georgios Pappas, M.D., Nikolaos Akritidis, M.D. etal. Brucellosis. N Engl J Med 2005; 352:2325-36.

4. Ruiz-Mesa JD, Sánchez-Gonzalez J, Reguera JM, Martín L, Lopez-Palmero S, Colmenero JD. Rose Bengal test: diagnostic yield and use for the rapid diagnosis of human brucellosis in emergency departments in endemic areas. Clin Microbiol Infect. 2005; 11: 221-5.

5. Jain R, Tilak V. Evaluation of different serological techniques in laboratory diagnosis of brucellosis. I Indian med Assoc. 2008; 106: 520-24

6. Mantur BG, Akki AS, Mangalgi SS, Patil SV, Gobbur RH, Peerpaur BV. Childhood brucellosis-- a microbiological, epidemiological and clinical study. J Trop Pediatr. 2004; 50: 153-7

7. Swai ES, schoonman L. Human brucellosis: seroprevalence and risk factors related to high risk occupational groups in Tanga Municipality, Tanzania. Zoonoses Public health. 2008; Epub ahead of print

8. Tabak F, Hakko E, mete B, Ozaras R, Mert A, Ozturk R. Is Family screening necessary in brucellosis? Infection. 2008; Epub ahead of print

9. John K, Kazwala R, Mfinanga GS. Knowledge of causes, clinical features and diagnosis of common zoonoses among medical practitioners in Tanzania. BMC infect Dis. 2008; Epub ahead of print

10. Queipo-ortuno MI, Colmenero JD, Bravo MJ, GarciaOrdonez MA, Morata P. Usefulness of a quantitative real rtimePCR assay using serum samples to discriminate between inactive, serologically positive and active human brucellosis. Clin Microbiol Infect 2008; Epub ahead of print 